\title{
SHARP WEAK TYPE INEQUALITIES FOR THE DYADIC MAXIMAL OPERATOR
}

\author{
ELEFTHERIOS N. NIKOLIDAKIS
}

\begin{abstract}
We obtain sharp estimates for the localized distribution function of $\mathcal{M} \phi$, when $\phi$ belongs to $L^{p, \infty}$ where $\mathcal{M}$ is the dyadic maximal operator. We obtain these estimates given the $L^{1}$ and $L^{q}$ norm, $q<p$ and certain weak- $L^{p}$ conditions.In this way we refine the known weak $(1,1)$ type inequality for the dyadic maximal operator.As a consequence we prove that the inequality

$$
\left\|\mathcal{M}_{\mathcal{T}} \phi\right\|_{p, \infty} \leq \frac{p}{p-1}\|\phi\|_{p, \infty}
$$

is sharp allowing every possible value for the $L^{1}$ and the $L^{q}$ norm for a fixed $q$ such that $1<q<p$, where $\|\cdot\|_{p, \infty}$ is the usual quasi norm on $L^{p, \infty}$.
\end{abstract}

Keywords: Dyadic, Maximal

\section{INTRODUCTION}

The dyadic maximal operator on $\mathbb{R}^{n}$ is defined by:

$$
\mathcal{M}_{d} \phi(x)=\sup \left\{\frac{1}{|Q|} \int_{Q}|\phi(u)| d u: x \in Q, Q \subseteq \mathbb{R}^{n} \text { is a dyadic cube }\right\}
$$

for every $\phi \in L_{l o c}^{1}\left(\mathbb{R}^{n}\right)$ where $|\cdot|$ is the Lebesgue measure on $\mathbb{R}^{n}$ and the dyadic cubes are those formed by the grids $2^{-N} \mathbb{Z}^{n}$ for $N=1,2, \ldots$.

As it is well known it satisfies the following weak type $(1,1)$ inequality

$$
\left|\left\{x \in \mathbb{R}^{n}: \mathcal{M}_{d} \phi(x) \geq \lambda\right\}\right| \leq \frac{1}{\lambda} \int_{\left\{\mathcal{M}_{d} \phi \geq \lambda\right\}}|\phi(u)| d u
$$

for every $\phi \in L^{1}\left(\mathbb{R}^{n}\right)$ and every $\lambda>0$ from which it is easy to get the following $L^{p}$ inequality:

$$
\left\|\mathcal{M}_{d} \phi\right\|_{p} \leq \frac{p}{p-1}\|\phi\|_{p}
$$

For every $p>1$ and $\phi \in L^{p}\left(\mathbb{R}^{n}\right)$ it is easy to see that the weak type inequality (1.3) is best possible and it is proved in [8] that (1.4) is also best possible (for general martingales see [2] and [3]).

In studying the dyadic maximal operator it would be convenient to work with functions supported in the unit cube $[0,1]^{n}$ and more generally defined on a non-atomic probability measure space $(X, \mu)$ where the dyadic sets are given in a family $\mathcal{T}$ of measurable subsets of $X$ that has a tree-like structure similar to the one in the dyadic case. Then we replace $\mathcal{M}_{d}$ by

$$
\mathcal{M}_{\mathcal{T}} \phi(x)=\sup \left\{\frac{1}{\mu(I)} \int_{I}|\phi| d \mu: x \in I \subseteq X, I \in \mathcal{T}\right\}
$$

and (1.3) and (1.4) remain true and sharp in this setting. 
Actually, in this general setting (1.4) has been improved even more by inserting the $L^{1}$-norm of $\phi$ as a variable giving the so called Bellman functions of the dyadic maximal operator. In fact in [4] the following function of variables $f, F$ has been explicitly computed

$$
B(f, F)=\sup \left\{\int_{X}\left(\mathcal{M}_{\mathcal{T}} \phi\right)^{p} d \mu: \phi \geq 0, \int_{X} \phi d \mu=f, \int_{X} \phi^{p} d \mu=F\right\}
$$

where $0<f^{p} \leq F$.

The related Bellman functions for the case $p<1$ have been also computed in [5].

It is interesting now to search what happens in case we replace the $L^{p}$-norm with the quasi norm $\|\cdot\|_{p . \infty}$ defined in $L^{p, \infty}$, where

$$
\|\phi\|_{p, \infty}=\sup \left\{\lambda \mu(\{|\phi| \geq \lambda\})^{1 / p}: \lambda>0\right\}
$$

for every $\phi$ such that this supremum is finite.

It is known that $L^{p, \infty}$ contains $L^{p}$ strictly and $\mathcal{M}_{\mathcal{T}}$ can be defined on $L^{p, \infty}$ with values on $L^{p, \infty}$. As a matter of fact it is not difficult to see that $\mathcal{M}_{\mathcal{T}}$ satisfies the following

$$
\left\|\mathcal{M}_{\mathcal{T}} \phi\right\|_{p, \infty} \leq \frac{p}{p-1}\|\phi\|_{p, \infty}
$$

for every $\phi \in L^{p, \infty}$.

In [7] it is proved that (1.8) is best possible.

Actually, a stronger fact is proved there, namely that

$$
\sup \left\{\left\|\mathcal{M}_{\mathcal{T}} \phi\right\|_{p, \infty}: \phi \geq 0, \int_{X} \phi d \mu=f,\|\phi\|_{p, \infty}=F\right\}=\frac{p}{p-1} F
$$

for every $(f, F)$ such that $0<f \leq \frac{p}{p-1} F$. That is (1.8) is sharp allowing every value for the $L^{1}$-norm of $\phi$.

In the present paper we precisely compute

$$
B(f, A, F, \lambda)=\sup \left\{\mu\left(\left\{\mathcal{M}_{\mathcal{T}} \phi \geq \lambda\right\}\right): \phi \geq 0, \int_{X} \phi d \mu=f, \int_{X} \phi^{q} d \mu=A,\|\phi\|_{p, \infty}=F\right\} .
$$

for a fixed $q$ such that $1<q<p$, and for all allowable values of $(f, A, F)$.In order to find (1.10) it is convenient to work with the following

$$
B_{1}(f, A, F, \lambda)=\sup \left\{\mu\left(\left\{\mathcal{M}_{\mathcal{T}} \phi \geq \lambda\right\}\right): \phi \geq 0, \int_{X} \phi d \mu=f, \int_{X} \phi^{q} d \mu=A,\|\phi\|_{p, \infty} \leq F\right\} .
$$

Our aim is to prove the following

Theorem 1.1. If $(f, A, F) \in D_{1}, A>A_{2}(f, F)$ then $B_{1}(f, A, F, \lambda)$ is given by

$$
B_{1}(f, A, F, \lambda)=\left\{\begin{array}{ll}
1, & \lambda \leq f \\
f / \lambda, & f<\lambda \leq \frac{p}{p-1} F \lambda_{3}^{\prime} \\
\delta^{\prime}, & \frac{p}{p-1} F \lambda_{3}^{\prime}<\lambda \leq \frac{p}{p-1} F \lambda_{1}^{\prime} \\
{\left[\frac{\frac{p}{p-1} / F}{\lambda}\right]^{p},} & \lambda<\frac{p}{p-1} F \lambda_{1}^{\prime}
\end{array}\right]
$$


where a) $\lambda_{1}^{\prime}$ is the unique solution of

$$
F_{\lambda^{\prime}}\left(\frac{1}{\left(\lambda^{\prime}\right)^{p}}\right)+\frac{\Gamma}{\left(\lambda^{\prime}\right)^{p-1}}=A
$$

for

$$
\lambda^{\prime}=\frac{p-1}{p} \lambda \frac{1}{F} \geq\left(\frac{1}{f_{1}}\right)^{1 /(p-1)}, \text { and } f_{1}=\frac{p-1}{p} \cdot \frac{f}{F} .
$$

b) $\lambda_{3}^{\prime}$ is the unique root of the equation

$$
T_{\lambda^{\prime}}\left(f_{1} / \lambda^{\prime}\right)=A \quad \text { on }\left[f_{1},\left(\frac{1}{f_{1}}\right)^{1 /(p-1)}\right]
$$

c) $\delta^{\prime}$ is the unique solution of $T_{\lambda^{\prime}}\left(\delta^{\prime}\right)+F_{\lambda^{\prime}}\left(\delta^{\prime}\right)=A$, for $\delta^{\prime} \in \Delta$, a suitable interval.

Here $T_{\lambda}$ and $F_{\lambda}$ are certain functions defined on appropriate intervals, and are defined in the sequel.

Additionally $D_{1}$ is the domain of the extremal problem defined as follows:

$$
\begin{aligned}
&(f, A, F) \in D_{1} \Leftrightarrow \text { i) } 0<f \leq \frac{p}{p-1} F \\
& \text { ii) } A_{2}(f, F) \leq A \leq A_{1}(f, F)
\end{aligned}
$$

where

$$
A_{1}(f, F)=\left(\frac{p-1}{p}\right)^{p-q / p-1} \frac{p}{p-q} f^{p-q / p-1} \cdot F^{p(q-1) /(p-1)}
$$

and

$$
A_{2}(f, F)= \begin{cases}f^{q}, & 0<f \leq F \\ F^{q} \frac{1}{p-q}\left\{p-q\left[p\left(1-\frac{p-1}{p} \cdot \frac{f}{F}\right)\right]^{p-q / p-1}\right\}, & F<f \leq \frac{p}{p-1} F .\end{cases}
$$

As a matter of fact we prove Theorem 1.1 in case where $F=\frac{p-1}{p}$. We state it at the beginning of Section 4 .

Actually our results generalize the results obtained in [6].

As an immediate Corollary we obtain

Theorem 1.2. The following holds

$$
\begin{gathered}
\sup \left\{\left\|\mathcal{M}_{\mathcal{T}} \phi\right\|_{p, \infty}: \phi \geq 0, \int_{X} \phi d \mu=f, \int_{X} \phi^{q} d \mu=A,\|\phi\|_{p, \infty}=F\right\} \\
=\frac{p}{p-1} F, \text { for every }(f, A, F) \in D_{1} .
\end{gathered}
$$

That is (1.9) is best possible allowing every value of the $L^{1}$ and $L^{q}$-norm.

At last we mention that all the above calculations are independent of the measure space and the associated tree. We begin now with: 


\section{Preliminaries}

Let $(X, \mu)$ be a non-atomic probability space.

The following holds:

Lemma 2.1. Let $\phi: X \rightarrow \mathbb{R}^{+}$be measurable and $I \subseteq X$ be measurable with $\mu(I)>0$. Suppose that $\frac{1}{\mu(I)} \int_{I} \phi d \mu=s$. Then for every $t$ such that $0<t \leq \mu(I)$ there exists a measurable set $E_{t} \subseteq I$ with $\mu\left(E_{t}\right)=t$ and $\frac{1}{\mu\left(E_{t}\right)} \int_{E_{t}} \phi d \mu=s$.

Proof. Consider the measure space $(I, \mu / I)$ and let $\psi: I \rightarrow \mathbb{R}^{+}$be the restriction of $\phi$ on $I$ that is $\psi=\phi / I$. Then if $\psi^{*}:(0, \mu(I)] \rightarrow \mathbb{R}^{+}$is the decreasing rearrangement of $\psi$, we have that

$$
\frac{1}{t} \int_{0}^{t} \psi^{*}(u) d u \geq \frac{1}{\mu(I)} \int_{0}^{\mu(I)} \psi^{*}(u) d u=s \geq \frac{1}{t} \int_{\mu(I)-t}^{\mu(I)} \psi^{*}(u) d u .
$$

Since $\psi^{*}$ is decreasing we get the inequalities in (2.12), while the equality is obvious since

$$
\int_{0}^{\mu(I)} \psi^{*}(u) d u=\int_{I} \phi d \mu .
$$

From (2.12) it is easily seen that there exists $r \geq 0$ such that $t+r \leq \mu(I)$ with

$$
\frac{1}{t} \int_{r}^{t+r} \psi^{*}(u) d u=s .
$$

It is also easily seen that there exists $E_{t}$ measurable subset of $I$ such that

$$
\mu\left(E_{t}\right)=t \text { and } \int_{E_{t}} \phi d \mu=\int_{r}^{t+r} \psi^{*}(u) d u
$$

since $(X, \mu)$ is non-atomic.

From (2.13) and (2.14) we get the conclusion of the lemma.

We now call two measurable subsets $A, B$ of $X$ almost disjoint if $\mu(A \cap B)=0$.

We give now the following

Definition 2.1. A set $\mathcal{T}$ of measurable subsets of $X$ will be called a tree if the following conditions are satisfied.

(i) $X \in \mathcal{T}$ and for every $I \in \mathcal{T}$ we have that $\mu(I)>0$.

(ii) For every $I \in \mathcal{T}$ there corresponds a finite or countable subset $C(I) \subseteq \mathcal{T}$ containing at least two elements such that:

(a) the elements of $C(I)$ are pairwise almost disjoint subsets of $I$.

(b) $I=\cup C(I)$.

(iii) $\mathcal{T}=\bigcup_{m \geq 0} \mathcal{T}_{(m)}$ where $\mathcal{T}_{0}=\{X\}$ and

$$
\mathcal{T}_{(m+1)}=\bigcup_{I \in \mathcal{T}_{(m)}} C(I)
$$

(iv) $\lim _{m \rightarrow+\infty} \sup _{I \in \mathcal{T}_{(m)}} \mu(I)=0$. 
From [4] we have the following

Lemma 2.2. For every $I \in \mathcal{T}$ and every $\alpha$ such that $0<\alpha<1$ there exists subfamily $\mathcal{F}(I) \subseteq \mathcal{T}$ consisting of pairwise almost disjoint subsets of I such that

$$
\mu\left(\bigcup_{J \in \mathcal{F}(I)} J\right)=\sum_{J \in \mathcal{F}(I)} \mu(J)=(1-\alpha) \mu(I)
$$

Let now $(X, \mu)$ be a non-atomic probability measure space and $\mathcal{T}$ a tree as in Definition 2.1. We define the associated maximal operator to the tree $\mathcal{T}$ as follows: For every $\phi \in L^{1}(X, \mu)$ and $x \in X$, then

$$
\mathcal{M} \phi(x)=\mathcal{M}_{\mathcal{T}} \phi(x)=\sup \left\{\frac{1}{\mu(I)} \int_{I}|\phi| d \mu: x \in I \in \mathcal{T}\right\}
$$

\section{Domain Of The Extremal Problem}

We prove the following:

Theorem 3.1. For $f$ and $A$ positive constants the following are equivalent

(i) There exists $\phi: X \rightarrow \mathbb{R}^{+} \mu$-measurable, non zero such that

$$
\int_{X} \phi d \mu=f, \quad \int_{X} \phi^{q} d \mu=A, \quad\|\phi\|_{p, \infty} \leq \frac{p-1}{p} .
$$

(ii) $0<f \leq 1$ and $A_{f} \leq A \leq \Gamma f^{p-q / p-=1}$ where $\Gamma=\left(\frac{p-1}{p}\right)^{q} \cdot \frac{p}{p-q}$ and $A_{f}$ is defined by:

$$
A_{f}= \begin{cases}f^{q}, & \text { if } 0<f \leq \frac{p-1}{p} \\ \left(\frac{p-1}{p}\right)^{q} \cdot \frac{1}{p-q}\left\{p-q[p(1-f)]^{p-q / p-1}\right\}, & \text { if } \quad \frac{p-1}{p}<f \leq 1 .\end{cases}
$$

(We say then that $(f, A) \in D$.).

Remark Actually the domain of the above theorem is the one defined in Theorem 1.1, for $F=\frac{p-1}{p}$. A scaling argument on Theorem 3.1 then gives the respective domain in Theorem 1.1 .

In order to prove the above theorem we will need the following.

Lemma 3.1. Let $g_{1}, g_{2}:[0,1] \rightarrow \mathbb{R}^{+}$be non-increasing functions satisfying the following property:

There exists $c \in(0,1)$ such that

$$
g_{1} \geq g_{2} \text { on }(0, c], \quad \text { and } g_{1} \leq g_{2} \text { on }(0,1] .
$$

Additionally, $\int_{0}^{1} g_{1}=\int_{0}^{1} g_{2}$. Then for every $q>1$ we have that $\int_{0}^{1} g_{1}^{q} \geq \int_{0}^{1} g_{2}^{q}$.

If in addition the set $\left\{g_{1}>g_{2}\right\} \cap(0, c]$ has positive Lesbesgue measure then

$$
\int_{0}^{1} g_{1}^{q}>\int_{0}^{1} g_{2}^{q}
$$


Lemma 3.1 is proved as soon as the following is proved. (A simple approximation argument gives the consequence).

Lemma 3.2. Let $\left(a_{i}\right)_{i=1}^{n}$, $\left(b_{i}\right)_{i=1}^{n}$ be finite $(n \geq 3)$, non-increasing sequences of non negative numbers satisfying the following property:

There exists $j \in[1, n) \cap \mathbb{N}$ such that

$$
\begin{aligned}
& b_{i} \geq a_{i}, \quad \forall i=1,2, \ldots, j \quad \text { and } \\
& b_{i} \leq a_{i}, \quad \forall i=j+1, \ldots, n .
\end{aligned}
$$

Additionally $a_{1}+a_{2}+\cdots+a_{n}=b_{1}+b_{2}+\cdots+b_{n}$. Then for every $q>1$ we have that

$$
b_{1}^{q}+b_{2}^{q}+\cdots+b_{n}^{q} \geq a_{1}^{q}+a_{2}^{q}+\cdots a_{n}^{q} .
$$

If in addition $b_{i}>a_{i}$ for some $i \in[1, j]$ then we have strict inequality in (3.15).

\section{Proof of Lemma 3.2}

We prove it by induction on $n \geq 3$.

For $n=3$, we have two sequences $\left(a_{1}, a_{2}, a_{3}\right)$ and $\left(b_{1}, b_{2}, b_{3}\right)$ such that

$$
a_{1} \geq a_{2} \geq a_{3} \geq 0 \text { and } b_{1} \geq b_{2} \geq b_{3} \geq 0, \text { with } a_{1}+a_{2}+a_{3}=b_{1}+b_{2}+b_{3} .
$$

Without loss of generality we also suppose, according to our hypothesis that

$$
b_{1} \geq a_{1}, \quad b_{2} \leq a_{2}, \quad b_{3} \leq a_{3} .
$$

We set $a=a_{1}+a_{2}+a_{3}$ and consider all $\left(x_{1}, x_{2}, x_{3}\right) \in \mathbb{R}^{3}$ such that $x_{i} \geq 0, \forall i=1,2,3$ and $x_{1} \geq a_{1}, x_{2} \leq a_{2}, x_{3} \leq a_{3}$ satisfying the additional condition $x_{1}+x_{2}+x_{3}=a$.

We then set

$$
\begin{gathered}
F\left(x_{1}, x_{2}\right)=x_{1}^{q}+x_{2}^{q}+\left(a-x_{1}-x_{2}\right)^{q} \text { with domain } \\
S=\left\{\begin{array}{c}
\left(x_{1}, x_{2}\right): x_{1} \geq a_{1}, x_{2} \leq a_{2}, 0 \leq x_{1}+x_{2} \leq a, \\
x_{i} \geq 0, i=1,2 .
\end{array}\right\}
\end{gathered}
$$

It is not difficult to see (by using the theory of Lagrange multipliers) that: $\min _{S} F=F\left(a_{1}, a_{2}\right)$, and that this minimum is attained in only one point, namely $\left(a_{1}, a_{2}\right)$. So the first step of the induction is easily proved.

We describe more extensively the inductive step.

We suppose that the Lemma holds for $n \in \mathbb{N}, n \geq 3$. We prove it for $n+1$. So we suppose that

$$
x_{1} \geq a_{1}, \quad x_{2} \geq a_{2}, \ldots, \quad x_{i} \geq a_{i}, \quad x_{i+1} \leq a_{i+1}, \ldots, x_{n} \leq a_{n}, \quad x_{n+1} \leq a_{n+1}
$$

for some $i \in\{1,2, \ldots, n\}$, and that

$$
x_{1}+x_{2}+\cdots+x_{n}+x_{n+1}=a_{1}+a_{2}+\cdots+a_{n}+a_{n+1} .
$$

We now set

$$
\begin{aligned}
& y_{j}=x_{j}, \quad 1 \leq j \leq n-1 \\
& y_{n}=x_{n}+x_{n+1} \\
& a_{j}^{\prime}=a_{j}, \quad 1 \leq j \leq n-1 \\
& a_{n}^{\prime}=a_{n}+a_{n+1} .
\end{aligned}
$$


We check now that

$$
a_{n}^{\prime} \geq y_{n} \text { or that } a_{n}+a_{n+1} \geq x_{n}+x_{n+1} .
$$

We distinguish two cases

A) If $a_{n} \geq x_{n}$ then since $a_{n+1} \geq x_{n+1}$, we have (3.16).

B) Let $a_{n}<x_{n}$, then by hypothesis we have that $a_{n+1} \geq x_{n+1}$ and $a_{i} \leq x_{i}, \forall i=1,2, \ldots, n$.

But $a_{1}+a_{1}+\cdots+a_{n}+a_{n+1}=x_{1}+x_{2}+\cdots+x_{n}+x_{n+1} \geq a_{1}+a_{2}+\cdots+a_{n-1}+x_{n}+x_{n+1} \Rightarrow(3.16)$.

So in any case (3.16) is true.

From the induction step we conclude that

$$
y_{1}^{q}+y_{2}^{q}+\cdots+y_{n}^{q} \geq\left(a_{1}^{\prime}\right)^{q}+\left(a_{2}^{\prime}\right)^{q}+\cdots+\left(a_{n}^{\prime}\right)^{q} .
$$

Then we have:

$$
\begin{aligned}
& x_{1}^{q}+x_{2}^{q}+\cdots+x_{n}^{q}+x_{n+1}^{q}-\left(a_{1}^{q}+a_{2}^{q}+\cdots+a_{n}^{q}+a_{n+1}^{q}\right) \\
= & \left(y_{1}^{q}+y_{2}^{q}+\cdots+y_{n-1}^{q}+y_{n}^{q}\right)-y_{n}^{q}+\left(x_{n}^{q}+x_{n+1}^{q}\right) \\
& -\left(\left(a_{1}^{\prime}\right)^{q}+\cdots+\left(a_{n}^{\prime}\right)^{q}\right)+\left(a_{n}^{\prime}\right)^{q}-a_{n}^{q}-a_{n+1}^{q} \\
= & {\left[\left(y_{1}^{q}+y_{2}^{q}+\cdots+y_{n}^{q}\right)-\left(\left(a_{1}^{\prime}\right)^{q}+\cdots+\left(a_{n}^{\prime}\right)^{q}\right)\right] } \\
& +\left[x_{n}^{q}+x_{n+1}^{q}+\left(a_{n}+a_{n+1}\right)^{q}-\left(\left(x_{n}+x_{n+1}\right)^{q}+a_{n}^{q}+a_{n+1}^{q}\right)\right] \\
= & \delta_{n}+\tau_{n} .
\end{aligned}
$$

Here $\delta_{n}=y_{1}^{q}+y_{2}^{q}+\cdots+y_{n}^{q}-\left(\left(a_{1}^{\prime}\right)^{q}+\cdots+\left(a_{n}^{\prime}\right)^{q}\right) \geq 0$ by (3.17) and so

$$
\begin{aligned}
x_{1}^{q}+\cdots+x_{n}^{q}+x_{n+1}^{q}-\left(a_{1}^{q}+\cdots+a_{n}^{q}+a_{n+1}^{q}\right) \geq \tau_{n}= & {\left[\left(a_{n}+a_{n+1}\right)^{q}+x_{n}^{q}+x_{n+1}^{q}\right] } \\
& -\left[a_{n}^{q}+a_{n+1}^{q}+\left(x_{n}+x_{n+1}\right)^{q}\right] .
\end{aligned}
$$

It is obvious now according to (3.16) that $a_{n}+a_{n+1} \geq x_{n} \geq x_{n+1}$, and no matter the sequence $\left(a_{n}, a_{n+1}, x_{n}+x_{n+1}\right)$ is arranged with $a_{n} \geq a_{n+1}$, we obtain two sequences $\left(a_{n}+a_{n+1}, x_{n}, x_{n+1}\right)$, $\left(a_{n}, a_{n+1}, x_{n}+x_{n+1}\right)$ which satisfy the hypothesis of the lemma in case where $n=3$. So we must have that $\tau_{n} \geq 0$. Additionally if there exists $j \in\{1,2, \ldots$,$\} such that x_{j}>a_{j}$ then either $j<n$ so that $\delta_{n}>0$ by the induction step, or $j=n$ and $x_{1}=a_{1}, \ldots, x_{n-1}=a_{n-1}$. In the latter case we must have that $x_{n}>a_{n}, x_{n+1}<a_{n+1} x_{n}+x_{n+1}=a_{n}+a_{n+1}$ and we easily prove that $x_{n}^{q}+x_{n+1}^{q}>a_{n}^{q}+a_{n+1}^{q}$. (This is in fact the case $n=2$, which is easy to handle).

We now prove the following

Theorem 3.2. Let $(\gamma, \delta]$ be a subinterval of $(0,1]$.

Then the following are equivalent

i) $\exists g:(\gamma, \delta] \rightarrow \mathbb{R}^{+}$non-increasing such that

$$
\begin{gathered}
g(t) \leq \psi(t)=\left(1-\frac{1}{p}\right) t^{-1 / p}, \quad \forall t \in(\gamma, \delta), \quad \text { and } \\
\int_{\gamma}^{\delta} g=f_{1}, \quad \int_{\gamma}^{\delta} g^{q}=A_{1} .
\end{gathered}
$$

ii) $0<f_{1} \leq \delta^{1-\frac{1}{p}}-\gamma^{1-\frac{1}{p}}$ and $z\left(f_{1}\right) \leq A_{1} \leq x\left(f_{1}\right)$, where $z\left(f_{1}\right), x\left(f_{1}\right)$ are defined below. 
Proof. i) $\Rightarrow$ ii) Suppose we are given $g$ with the conditions in i). Let

$$
\begin{gathered}
f=\int_{\gamma}^{\delta} \psi=\delta^{1-\frac{1}{p}}-\gamma^{1-\frac{1}{p}} \\
A=\int_{\gamma}^{\delta} \psi^{q}=\Gamma\left(\delta^{1-\frac{q}{p}}-\gamma^{1-\frac{q}{p}}\right), \quad \text { where } \\
\Gamma=\left(\frac{p-1}{p}\right)^{q} \frac{p}{p-q} .
\end{gathered}
$$

Since $g \leq \psi$ on $(\gamma, \delta]$ we must have

$$
f_{1} \leq f, \quad A_{1} \leq A
$$

Consider now the following function defined on $(\gamma, \delta], g_{1}(t)=\left\{\begin{array}{cc}\psi(t), & t \in(\gamma, c] \\ 0, & t \in(c, \delta],\end{array}\right.$, where $c$ is such that

$$
\int_{\gamma}^{\delta} g_{1}=f_{1}
$$

Then from (3.18) we can easily see that $c=\left(\gamma^{1-\frac{1}{p}}+f_{1}\right)^{p / p-1}$. Then

$$
\int_{\gamma}^{\delta} g_{1}^{q}=\Gamma\left\{\left(\gamma^{1-\frac{1}{p}}+f_{1}\right)^{p-q / p-1}-\gamma^{1-\frac{q}{p}}\right\}=: x\left(f_{1}\right) .
$$

Given the above $g:(\gamma, \delta] \rightarrow \mathbb{R}^{+}$we easily see that the requirements for Lemma 3.1 for the function $g, g_{1}$ are satisfied, so we must have

$$
\int_{\gamma}^{\delta} g^{q} \leq \int_{\gamma}^{\delta} g_{1}^{q}=: x\left(f_{1}\right) \Rightarrow A_{1} \leq x\left(f_{1}\right)
$$

that is what we wanted to prove.

We consider now two cases:

a) $0<f_{1}<(\delta-\gamma) \delta^{-\frac{1}{p}} \cdot \frac{p-1}{p}=(\delta-\gamma) \psi(\delta)$.

We then must have that

$$
\left(\int_{\gamma}^{\delta} g\right)^{q} \leq(\delta-\gamma)^{q-1}\left(\int_{\gamma}^{\delta} g^{q}\right),
$$

in view of Holder's inequality, that is $f_{1}^{q} \leq(\delta-\gamma)^{q-1} A_{1} \Rightarrow A_{1} \geq \frac{f_{1}^{q}}{(\delta-\gamma)^{q-1}}$.

b) $(\delta-\gamma) \frac{p-1}{p} \delta^{-1 / p}<f_{1} \leq f=\left(\delta^{1-\frac{1}{p}}-\gamma^{1-\frac{1}{p}}\right)$.

Then we consider the following function $g_{2}:(\gamma, \delta] \rightarrow \mathbb{R}^{+}$such that

$$
g_{2}(t)=\left\{\begin{array}{cc}
\left(1-\frac{1}{p}\right) c^{-1 / p}, & t \in(\gamma, c] \\
\psi(t), & t \in(c, \delta]
\end{array}\right.
$$

with $c$ such that $\int_{\gamma}^{\delta} g_{2}=f_{1}$. It is easy to see then that $c$ must satisfy:

$$
\frac{1}{p} c^{1-\frac{1}{p}}+\gamma \frac{p-1}{p} c^{-1 / p}=\delta^{1-\frac{1}{p}}-f_{1}
$$


It is, in fact, easy to see that there exists unique $c$ satisfying (3.19) as soon as $f_{1}$ is described as in case b) above. Then for that $c$ we obtain

$$
\int_{\gamma}^{\delta} g_{2}^{q}=(c-\gamma)\left(\frac{p-1}{p}\right)^{q} c^{-q / p}+\Gamma\left(\delta^{1-\frac{q}{p}}-c^{1-\frac{q}{p}}\right)=: y\left(f_{1}\right)
$$

where $c:=c\left(f_{1}\right)$ as in (3.19).

Then using again Lemma 3.1 for $g$ and $g_{2}$ we must have that $A_{1}=\int_{\gamma}^{\delta} g^{q} \geq y\left(f_{1}\right)$. Defining now

$$
z\left(f_{1}\right)=\left\{\begin{array}{cc}
f_{1}^{q} /(\delta-\gamma)^{q-1}, & 0<f_{1}<(\delta-\gamma) \delta^{-1 / p} \cdot \frac{p-1}{p} \\
y\left(f_{1}\right), & (\delta-\gamma) \frac{p-1}{p} \delta^{-1 / p} \leq f_{1} \leq\left(\delta^{1-\frac{1}{p}}-\gamma^{1-\frac{1}{p}}\right)
\end{array}\right.
$$

we must have that

$$
z\left(f_{1}\right) \leq A_{1}=\int_{)}^{\delta} \gamma g^{q} \leq x\left(f_{1}\right)
$$

that is what we wanted to prove

ii) $\Rightarrow$ i) Suppose now that we are given $f_{1}, A_{1}$ :

$$
\begin{aligned}
& 0<f_{1} \leq \delta^{1-\frac{1}{p}}-\gamma^{1-\frac{1}{p}} \\
& z(h) \leq A_{1} \leq x\left(f_{1}\right) .
\end{aligned}
$$

In the proof of i) $\Rightarrow$ ii) we saw that there exist functions $h_{1}, h_{2}:(\gamma, \delta] \rightarrow \mathbb{R}^{+}$non-increasing such that $\int_{\gamma}^{\delta} h_{1}=\int_{\gamma}^{\delta} h_{2}=f_{1}$ and

$$
\int_{\gamma}^{\delta} h_{1}^{q}=x\left(f_{1}\right), \quad \int_{\gamma}^{\delta} h_{2}^{q}=z\left(f_{1}\right)
$$

with $h_{1}(t)_{1}, h_{2}(t) \leq \psi(t)$, for every $t \in(\gamma, \delta]$.

Consider now the function

$$
\begin{gathered}
T:[0,1] \rightarrow \mathbb{R}^{+} \quad \text { such that } \\
T(\ell)=\int_{\gamma}^{\delta}\left[\ell h_{1}+(1-\ell) h_{2}\right]^{q} .
\end{gathered}
$$

It is obvious that $T$ is continuous on $[0,1]$ and that $T(0)=z\left(f_{1}\right)$ while $T(1)=x\left(f_{1}\right)$.

As a result there exists $\ell \in[0,1]$ such that $\int_{\gamma}^{\delta} h_{\ell}^{q}=A_{1}$, where $h_{\ell}=\ell h_{1}+(1-\ell) h_{2}$. Additionally $\int_{\gamma}^{\delta} h_{\ell}=f_{1}$ so $h_{\ell}$ is the wanted function, satisfying the conditions of i).

As a corollary we obtain the following propositions.

Proposition 3.1. Let $\alpha \in(0,1] f_{1}, A_{1}$ positive numbers, were $f_{1} \leq \alpha^{1-\frac{1}{p}}$. Then the following are equivalent

(i) $\exists g:(0, \alpha] \rightarrow \mathbb{R}^{+}$Lebesgue measurable such that $g \leq \psi$ on $(0, a]$ and

$$
\int_{0}^{\alpha} g=f_{1}, \quad \int_{0}^{\alpha} g^{q}=A_{1} .
$$


(ii) a) If $0<f_{1} \leq \frac{p-1}{p} \alpha^{1-\frac{1}{p}}$ then $\frac{f_{1}^{q}}{\alpha^{q-1}} \leq A_{1} \leq \Gamma f_{1}^{p-q / p-1}$

b) If $\frac{p-1}{p} \alpha^{1-\frac{1}{p}} \leq f_{1} \leq \alpha^{1-\frac{1}{p}}$ then

$$
\Delta_{f_{1}}(\alpha) \leq A_{1} \leq \Gamma f_{1}^{\frac{p-q}{p-1}}
$$

where

$$
\left.\Delta_{f_{1}}(\alpha)=\left(\frac{p-1}{p}\right)^{p} \frac{1}{p-q}\left\{p \alpha^{1-\frac{q}{p}}-q\left[p\left(\alpha^{1-\frac{1}{p}}-f_{1}\right)\right]\right]^{p-q / p-1}\right\}
$$

Proposition 3.2. For $a \in(0,1]$ and $f_{2}, A_{2}$ such that $f_{2} \leq 1-\alpha^{1-\frac{1}{p}}$ the following are equivalent

(i) $\exists g:[a, 1] \rightarrow \mathbb{R}^{+}$Lebesgue measurable such that

$$
g \leq \psi \quad \text { on }[\alpha, 1] \text { and } \quad \int_{\alpha}^{1} g=f_{2}, \int_{\alpha}^{1} g^{q}=A_{2} .
$$

(ii) a) If $f_{2} \leq(1-\alpha) \frac{p-1}{p}$ then $\frac{f_{2}^{q}}{(1-\alpha)^{q-1}} \leq A_{2} \leq E_{f_{2}}(\alpha)$ where

$$
E_{f_{2}}(\alpha)=\Gamma\left[\left(f_{2}+\alpha^{1-\frac{1}{p}}\right)^{p-q / p-1}-\alpha^{1-\frac{q}{p}}\right] .
$$

b) If $(1-\alpha) \frac{p-1}{p} \leq f_{2} \leq 1-\alpha^{1-\frac{1}{p}}$ then

$$
\Gamma_{f_{2}}(\alpha) \leq A_{2} \leq E_{f_{2}}(\alpha)
$$

where

$$
\Gamma_{f_{2}}(\alpha)=\left(\frac{p-1}{p}\right)^{q} c^{-q / p}(c-\alpha)+\Gamma\left(1-c^{1-\frac{q}{p}}\right)
$$

and c satisfies

$$
\frac{1}{p} c^{1-\frac{1}{p}}+\left(1-\frac{1}{p}\right) \alpha c^{-1 / p}=1-f_{2}
$$

As a consequence, setting $a=1$ in Proposition 3.1 we obtain Theorem 3.1,

Using the above arguments one can easily prove that the following is true:

Theorem 3.3. For $f$ and $A$ positive constants with $A \neq f^{q}$ the following are equivalent:

(i) There exists $\phi:(X, \mu) \rightarrow \mathbb{R}^{+}$measurable, non zero, such that

$$
\int_{X} \phi d \mu=f, \quad \int_{X} \phi^{q} d \mu=A, \quad\|\phi\|_{p, \infty}=\frac{p-1}{p} .
$$

(ii) $0<f \leq 1$ and $A_{f} \leq A \leq \Gamma f^{p-q / p-1}$. 


\section{The Extremal Problem}

We state now Theorem 1.1 in case where $F=\frac{p-1}{p}$.

Theorem 4.1. Let $(f, A) \in D, A>A_{f}$ and $B_{1}(f, A, \lambda)$ given by

$$
\begin{array}{r}
B_{1}(f, A, \lambda)=\sup \left\{\mu\left(\left\{\mathcal{M}_{\mathcal{T}} \phi \geq \lambda\right\}\right): \phi \geq 0, \int_{X} \phi d \mu=f, \int_{X} \phi^{q} d \mu=f,\right. \\
\left.\|\phi\|_{p, \infty}=\frac{p-1}{p}\right\},
\end{array}
$$

Then the following hold

$$
B_{1}(f, A, \lambda)=\left\{\begin{array}{cc}
1, & 0<\lambda<f \\
\frac{f}{\lambda}, & f \leq \lambda<\lambda_{3} \\
\delta, & \lambda_{3} \leq \lambda<\lambda_{1} \\
\frac{1}{\lambda^{p}}, & \lambda_{1}<\lambda
\end{array}\right.
$$

where $\delta$ is the unique root of the equation $F_{\lambda}(\gamma)+T_{\lambda}(\gamma)=A, \lambda_{3}$ the unique element of the interval $\left[f,\left(\frac{1}{f}\right)^{1 / p-1}\right]$ such that $T_{\lambda}(f / \lambda)=A$ and $\lambda_{1}$ the unique root of the equation $F_{\lambda}\left(1 / \lambda^{p}\right)+\frac{\Gamma}{\lambda^{p-q}}=A$ on the interval $\left[\left(\frac{1}{f}\right)^{1 / p-1},+\infty\right)$.

Here $T_{\lambda}, F_{\lambda}$ are certain functions defined on appropriate intervals in the sequel.

In this section we are going to prove the following

Theorem 4.2. If $\alpha=\alpha(\lambda)=B_{1}(f, A, \lambda)$ where $(f, A) \in D$ with $A_{f}<A$, then

(i) $\alpha(\lambda)=\frac{1}{\lambda^{p}}$ for every $\lambda \geq \lambda_{1}$, where $\lambda_{1}$ is the unique root of the equation

$$
F_{\lambda}\left(\frac{1}{\lambda^{p}}\right)+\frac{\Gamma}{\lambda^{p-q}}=A \text { on the interval }\left(\left(\frac{1}{f}\right)^{1 / p-1},+\infty\right) \text {. }
$$

(ii) For every $f<\lambda<\lambda_{1}$, $\alpha$ equals the supremum of all $\beta$ such that $\beta \leq \min \left\{\frac{f}{\lambda}, \frac{1}{\lambda^{p}}\right\}$ and $f-\lambda \beta \leq 1-\beta^{1-\frac{1}{p}}$ for which $T_{\lambda}(\beta) \leq A-F_{\lambda}(\beta) \leq S_{\lambda}(\beta)$.

Proof. Let $\phi$ be as in (4.21). We work out the case $\lambda>\frac{p-1}{p}$. A few comments follow for the rest range of $\lambda$ 's.

Fix a $\lambda>\frac{p-1}{p}$. We set $E=\left\{\mathcal{M}_{\mathcal{T}} \phi \geq \lambda\right\}$.

Then $E$ is the almost disjoint union of elements of $\mathcal{T}, I_{j}, j=1,2, \ldots$. Indeed, we just need to consider those $I \in \mathcal{T}$ maximal under the condition $\frac{1}{\mu(I)} \int_{I} \phi d \mu \geq \lambda$.

For every $j$ we have that

$$
\int_{I_{j}} \phi d \mu \geq \lambda \mu\left(I_{j}\right)
$$

Summing the above equations up to $j$ we get

$$
\int_{E} \phi d \mu \geq \lambda \mu(E)
$$


We again consider the decreasing rearrangement of $\phi$, let $\phi^{*}:(0,1] \rightarrow \mathbb{R}^{+}$. In this point we need a fact which is true on every non-atomic finite measure space and can be seen in [1].

Namely that for every $\delta \in[0,1]$

$$
\int_{0}^{\delta} \phi^{*}(t) d t=\sup \left\{\begin{array}{ll}
\int_{K} \phi d \mu: & K \text { measurable subset } \\
\text { of } X \text { such that } \mu(K)=\delta
\end{array}\right\}
$$

where the supremum is actually attained.

From (4.26) we now get in view of the previous comment for $a=\mu(E)$ that $\int_{0}^{a} \phi^{*}(t) d t \geq \lambda a$, so if we define by

$$
T(f, A, \lambda)=\sup \left\{\begin{array}{ll} 
& \exists g:(0,1] \rightarrow \mathbb{R}^{+} \text {decreasing } \\
\alpha \in(0,1]: & \text { such that } \int_{0}^{1} g=f, \int_{0}^{1} g^{q}=A, g \leq \psi \\
\text { and } \int_{0}^{\alpha} g \geq \alpha \lambda
\end{array}\right\}
$$

we have that

$$
B_{1}(f, A, \lambda) \leq T(f, A, \lambda) .
$$

In fact in relation (4.23) the converse inequality is also true. To prove this we need the following.

Lemma 4.1. If $a \in(0,1]$ and $g:(0,1] \rightarrow \mathbb{R}^{+}$are such that

$$
\int_{0}^{a} g(t) d t \geq a \lambda, \quad \int_{0}^{1} g(t) d t=f, \int_{0}^{1} g^{q}(t) d t=A, \quad g^{*} \leq \psi
$$

then there exists $\phi:(X, \mu) \rightarrow \mathbb{R}^{+}$measurable with

$$
\int_{X} \phi d \mu=f, \int_{X} \phi^{q} d \mu=A,\|\phi\|_{p, \infty} \leq \frac{p-1}{p}
$$

with the additional property:

$$
\mu(\{\mathcal{M} \phi \geq \lambda\}) \geq a .
$$

Proof. Indeed from Lemma 2.2 by setting $I=X$ we guarantee the existence of a sequence $\left(I_{j}\right)_{j}$ of pairwise almost disjoint elements of $\mathcal{T}$ in such a way that

$$
\mu\left(\bigcup_{j} I_{j}\right)=\sum \mu\left(I_{j}\right)=a
$$

We consider the measure space $((0, a],|\cdot|)$ where $|\cdot|$ is Lebesgue measure. Because of $\int_{0}^{a} g(t) d t \geq$ $a \lambda$, applying Lemma 2.1 repeatedly we have as a consequence the existence of a partition $S=\left\{A_{j}, j=1,2, \ldots\right\}$ of $(0, a]$, which consists of Lebesgue measurable subsets of $(0, a]$ such that

$$
\left|A_{j}\right|=\mu\left(I_{j}\right) \text { and } \int_{A_{j}} g(t) d t \geq \lambda\left|A_{j}\right|
$$

For every $j=1,2, \ldots$ let now $g_{j}=\left(g / A_{j}\right)^{*}$ defined on $\left(0,\left|A_{j}\right|\right]$. Since $(X, \mu)$ is non-atomic and $\mu\left(I_{j}\right)=\left|A_{j}\right|$ we easily see that for every $j$ there exists $\phi_{j}: I_{j} \rightarrow \mathbb{R}^{+}$measurable such that $\phi_{j}^{*}=g_{j}$. Additionally suppose that $g^{\prime}=(g /(a, 1])^{*}$ and set $Y=X \backslash \cup I_{j}$. Since $\mu(Y)=1-a$ for the same reasons we get a $\phi^{\prime}: Y \rightarrow \mathbb{R}^{+}$such that $\left(\phi^{\prime}\right)^{*}=g^{\prime}$. Then since $I_{j}$ are pairwise 
almost disjoint there exists a measurable function $\phi: X \rightarrow \mathbb{R}^{+}$such that $\left.\phi\right|_{I_{j}}=\phi_{j}$ almost everywhere for every $j$ and $\left.\phi\right|_{Y}=\phi^{\prime}$. Then it is easy to see that

$$
\phi^{*}=g^{*} \leq \psi, \quad \int_{I_{j}} \phi d \mu=\int_{A_{j}} g d \mu \geq \lambda\left|A_{j}\right|=\lambda \mu\left(I_{j}\right)
$$

where the first equality holds almost everywhere with respect to the Lesbesgue measure. As a consequence we have that the norm (or quasi norm) condtitions for $\phi$ are satisfied and that

$$
\frac{1}{\mu\left(I_{j}\right)} \int_{I_{j}} \phi d \mu \geq \lambda \text { for every } j=1,2, \ldots
$$

So, $\{\mathcal{M} \phi \geq \lambda\} \supseteq \cup I_{j}$. As a consequence we get $\mu(\{\mathcal{M} \phi \geq \lambda\}) \geq a$ and the lemma is proved.

We continue now the proof of Theorem 4.1. Using Lemma 4.1 for $g$ non-increasing we see that

$$
B_{1}(f, A, \lambda)=T(f, A, \lambda)
$$

In fact we have equality in (4.26) even if we replace the inequality $\int_{0}^{a} g(t) d t \geq \alpha \lambda$, given in the definition of $T(f, A, \lambda)$ by equality, thus getting the function $S(f, A, \lambda)$ defined by

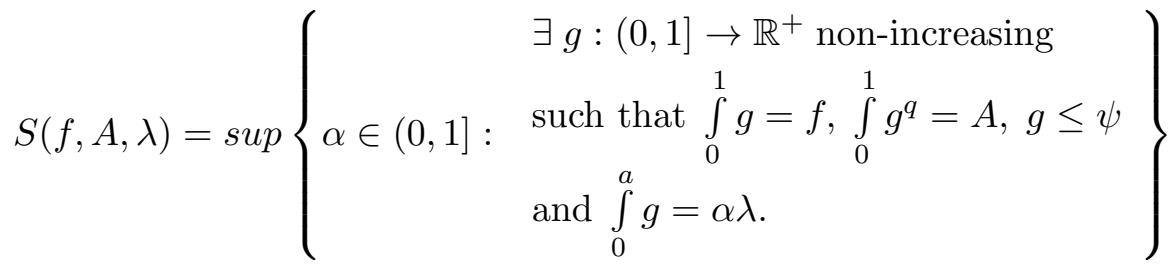

This is true because if $g$ is as in the definition of $T(f, A, \lambda)$, then of course $\int_{0}^{a} g(t) d t \geq \alpha \lambda$. But then there exists $\beta \geq \alpha$ such that $\int_{0}^{\beta} g(t) d t=\beta \lambda$ since $\theta(t)$ is a $\mathrm{n}$ on-increasing function of $t$, where $\theta$ is defined by $\theta:(0,1] \rightarrow \mathbb{R}^{+}$with $\theta(t)=\frac{1}{t} \int_{0}^{t} g(u) d u$ ( $g$ is non-increasing). Then we just need to apply Lemma 4.1 with $\beta$ in place of $\alpha$.

From the above we obtain that $B_{1}(f, A, \lambda)$ equals the supremum of all $\alpha \in(0,1]$ for which there exists $g:(0,1] \rightarrow \mathbb{R}^{+}$non-increasing and $A_{1}, A_{2} \geq 0$ such that

$$
\int_{0}^{a} g=f, \int_{0}^{a} g^{q}=A_{1}, \int_{\alpha}^{1} g=f_{2}, \int_{\alpha}^{1} g^{q}=A_{2}
$$

and $g \leq \psi$ where $A_{1}+A_{2}=A, f_{1}=\lambda \alpha, f_{2}=f-\lambda \alpha$.

We now take advantage the Proposition 3.1 and 3.2 of Section 3.

In light of the above mentioned fact and Proposition 3.1 for a fixed $\lambda>f$ and $\lambda>\frac{p-1}{p}$ we define the following functions

$$
T_{\lambda}, S_{\lambda}:\left[0,1 / \lambda^{p}\right] \rightarrow \mathbb{R}^{+} \text {by }
$$




$$
T_{\lambda}(a)=\left\{\begin{array}{ccc}
\lambda^{q} \alpha, & \text { for } & \alpha \leq\left[\frac{p-1}{p} / \lambda\right]^{p} \\
\Delta_{f_{1}}(\alpha), & \text { for } & {\left[\frac{p-1}{p} / \lambda\right]^{p}<\alpha \leq \frac{1}{\lambda^{p}}}
\end{array}\right.
$$

where $f_{1}=\lambda \alpha$ and $S_{\lambda}(\alpha)=\Gamma(\lambda \alpha)^{(p-q) /(p-1)}$.

In light now of Proposition 3.2 we also define:

$$
F_{\lambda}, G_{\lambda}:[0, f / \lambda] \rightarrow \mathbb{R}^{+} \text {for } \alpha \text { such that } f-\lambda \alpha \leq 1-\alpha^{1-\frac{1}{p}}
$$

a) If $0<f \leq \frac{p-1}{p}$

$$
F_{\lambda}(\alpha)=\frac{(f-\lambda \alpha)^{q}}{(1-\alpha)^{q-1}} \text { and } G_{\lambda}(\alpha)=E_{f_{2}}(\alpha)
$$

b) while if $\frac{p-1}{p}<f \leq 1$

$$
F_{\lambda}(\alpha)=\left\{\begin{array}{ccc}
\frac{(f-\lambda \alpha)^{q}}{(1-\alpha)^{q-1}}, & \text { for } & \frac{f-\frac{p-1}{p}}{\lambda-\frac{p-1}{p}} \leq \alpha \leq \frac{f}{\lambda} \\
\Gamma_{f_{2}}(\alpha), & \text { for } & \alpha \leq \frac{f-\frac{p-1}{p}}{\lambda-\frac{p-1}{p}}
\end{array}\right.
$$

and $G_{\lambda}(\alpha)=E_{f_{2}}(\alpha)$, where $f_{2}=f-\lambda \alpha$.

After giving the definitions of $T_{\lambda}, S_{\lambda}, F_{\lambda}, G_{\lambda}$ we can say that $B_{1}(f, A, \lambda)$ equals the supremum of all $\alpha \in(0,1]$ such that $\alpha \leq \min \left\{\frac{f}{\lambda}, \frac{1}{\lambda^{p}}\right\}$ and $f-\lambda \alpha \leq 1-\alpha^{1-\frac{1}{p}}$ for which there exist $A_{1}, A_{2} \geq 0$ with

$$
\left.\begin{array}{l}
T_{\lambda}(\alpha) \leq A_{1} \leq S_{\lambda}(\alpha) \\
F_{\lambda}(\alpha) \leq A_{2} \leq G_{\lambda}(\alpha)
\end{array}\right\} \quad \text { and } \quad A=A_{1}+A_{2}
$$

We now state the following

Lemma 4.2. If $(f, A) \in D$ and $\lambda>f$ such that $\alpha=\alpha(\lambda)=B_{1}(f, A, \lambda)<\frac{1}{\lambda^{p}}$ then there exists $g:(0,1] \rightarrow \mathbb{R}^{+}$such that $g \leq \psi, \int_{0}^{\alpha} g=\alpha \lambda, \int_{0}^{1} g=f, \int_{0}^{1} g^{q}=A$ and $\int_{\alpha}^{1} g^{q}=A_{2}$ where $A_{2}=F_{\lambda}(\alpha)$.

Proof. From the definition of $\alpha(\lambda)=\alpha$ and Corollary 4.2 it follows that there exists $g:(0,1] \rightarrow \mathbb{R}^{+}$with $g \leq \psi, \int_{0}^{1} g=f, \int_{0}^{\alpha} g=\lambda \alpha, \int_{0}^{\alpha} g^{q}=A_{1}, \int_{\alpha}^{1} g^{q}=A_{2}$, where $A_{1}+A_{2}=A$ and $F_{\lambda}(\alpha) \leq A_{2} \leq G_{\lambda}(\alpha)$. In fact, we prove that for every such $g$ we have that $A_{2}=F_{\lambda}(\alpha)$.

Suppose $g$ is as above with $A_{2}>F_{\lambda}(\alpha)$. Then $|\{g<\psi\} \cap(0, \alpha]| \geq \delta>0$. This is true since if $g=\psi$ on $(0, \alpha]$ then

$$
\int_{0}^{\alpha} g=\int_{0}^{\alpha} \psi=\alpha^{1-\frac{1}{p}}>\lambda \alpha, \text { since } \alpha<1 / \lambda^{p} .
$$

But, then there exists $\beta>\alpha$ such that $\int_{0}^{\beta} g \geq \beta \lambda$ which gives from the definition of $\alpha(\lambda)$ that $\alpha=\alpha(\lambda) \geq \beta$ a contradiction.

Let now $\varepsilon>0$ be small enough (we will arrange it's choice at the end), with $A_{2}-\varepsilon>F_{\lambda}(\alpha)$.

Since $F_{\lambda}(\alpha)<A_{2}-\varepsilon<G_{\lambda}(\alpha)$, by Proposition 4.2 there exists $g_{1}:[\alpha, 1] \rightarrow \mathbb{R}^{+}$such that $g_{1} \leq \psi, \int_{\alpha}^{1} g_{1}=f-\lambda \alpha, \int_{\alpha}^{1} g_{2}^{q}=A_{2}-\varepsilon$. We set $g_{1}=g$ on $(0, \alpha]$.

Since now $\left|\left\{g_{1}<\psi\right\} \cap(0, \alpha]\right| \geq \delta>0$ we can construct a function $g_{2}:(0,1] \rightarrow \mathbb{R}^{+}$with the same integral as $g_{1}$ and larger $L^{q}$-norm. This is done by increasing it's values on $(0, \alpha]$ and decreasing in appropriate way it's values on $[\alpha, 1]$, so that $g_{2} \leq \psi$. If $\varepsilon>0$ small enough we 
can arrange everything (since $\delta$ is fixed positive) in such a way that $\int_{0}^{1} g_{2}^{q}=A$. Additionally we have that $\int_{0}^{1} g_{2}=f$ and $\int_{0}^{\alpha} g_{2}>\lambda \alpha$, because we have increased the values of $g_{1}$ to $g_{2}$ in the interval $(0, \alpha]$. This gives as before the existence of a $\beta>\alpha$ such that $\int_{0}^{\beta} g_{2} \geq \lambda \beta$, so $\alpha(\lambda) \geq \beta>\alpha$, a contradiction.

In the sequel we state and prove the following

Lemma 4.3. For $(f, A) \in D$ such that $A>\mathcal{A}_{f}$ and $\alpha(\lambda)=B_{1}(f, A, \lambda)$, there exists $\lambda_{1} \geq$ $\left(\frac{1}{f}\right)^{1 / p-1}$ such that $\alpha(\lambda)=\frac{1}{\lambda^{p}}$, for every $\lambda \geq \lambda_{1}$.

Proof. If $\lambda \geq\left(\frac{1}{f}\right)^{1 / p-1}$ then $\frac{1}{\lambda^{p}} \leq \frac{f}{\lambda}$.

We consider the equation

We easily see that

$$
F_{\lambda}\left(1 / \lambda^{p}\right)=A-\frac{\Gamma}{\lambda^{p-q}} .
$$

$$
\lim _{\lambda \rightarrow \infty} F_{\lambda}\left(1 / \lambda^{p}\right)=\mathcal{A}_{f}<A=\lim _{\lambda \rightarrow+\infty}\left(A-\frac{\Gamma}{\lambda^{p-q}}\right) .
$$

For $\lambda=\lambda_{0}=\left(\frac{1}{f}\right)^{1 / p-1}$ we have that

$$
F_{\lambda_{0}}\left(1 / \lambda_{0}^{p}\right)=F_{\lambda_{0}}\left(\frac{f}{\lambda_{0}}\right)=0 \geq A-\Gamma f^{p-q / p-1}=A-\frac{\Gamma}{\lambda_{0}^{p-q}} .
$$

So, there exists $\lambda \geq \lambda_{0}$ such that

$$
F_{\lambda}\left(1 / \lambda^{p}\right)=A-\frac{\Gamma}{\lambda^{p-q}} .
$$

Let

$$
\lambda_{1}=\inf \left\{\lambda \geq \lambda_{0}: F_{\lambda}\left(1 / \lambda^{p}\right)=A-\frac{\Gamma}{\lambda^{p-q}}\right\}
$$

which is obviously a minimum. Then

$$
F_{\lambda_{1}}\left(\frac{1}{\lambda_{1}^{p}}\right)=A-\frac{\Gamma}{\lambda_{1}^{p-q}} .
$$

Consider the interval $\left[\frac{1}{\lambda_{1}^{p}}, 1\right]$. Applying Proposition 3.2 for $\alpha=\frac{1}{\lambda_{1}^{p}}, f_{2}=f-\frac{1}{\lambda_{1}^{p-1}}$ we obtain that there exists $g_{2}:\left[\frac{1}{\lambda_{1}^{p}}, 1\right] \rightarrow \mathbb{R}^{+}$such that

$$
g_{2} \leq \psi /\left[\frac{1}{\lambda_{1}^{p}, 1}\right], \int_{1 / \lambda_{1}^{p}}^{1} g_{2}=f-\frac{1}{\lambda_{1}^{p-1}}, \int_{1 / \lambda_{1}^{p}}^{1} g_{2}^{q}=F_{\lambda_{1}}\left(\frac{1}{\lambda_{1}^{p}}\right) .
$$

But then if $g:(0,1] \rightarrow \mathbb{R}^{+}$with $g /_{\left(0, \frac{1}{\lambda_{1}^{p}}\right]}=\psi$ and $g /{ }_{\left[\frac{1}{\lambda_{1}^{p}}, 1\right]}=g_{2}$ we have because of the above that

$$
\int_{0}^{1} g_{1}=f, \int_{0}^{1} g^{q}=A, g \leq \psi, \int_{0}^{1 / \lambda_{1}^{p}} g=\frac{1}{\lambda_{1}^{p-1}}=\frac{1}{\lambda_{1}^{p}} \cdot \lambda_{1}
$$


and according to Lemma4.1 we have that $\alpha\left(\lambda_{1}\right)=B_{1}\left(f, A, \lambda_{1}\right) \geq \frac{1}{\lambda_{1}^{p}}$. But of course $\alpha\left(\lambda_{1}\right) \leq \frac{1}{\lambda_{1}^{p}}$, so that $\alpha\left(\lambda_{1}\right)=\frac{1}{\lambda_{1}^{p}}$. Then we easily see that $\alpha(\lambda)=\frac{1}{\lambda^{p}}$ for every $\lambda \geq \lambda_{1}$. This is true because $g:(0,1] \rightarrow \mathbb{R}^{+}$as mentioned before satisfies:

$$
g \leq \psi, \int_{0}^{1 / \lambda^{p}} g=\frac{1}{\lambda^{p-1}}=\frac{1}{\lambda^{p}} \cdot \lambda
$$

for every such $\lambda$ and $\int_{0}^{1} g=f, \int_{0}^{1} g^{q}=A$,for every such $\lambda$.

Let now $\lambda_{2}=\min \left\{\lambda: \alpha(\lambda)=\frac{1}{\lambda^{p}}\right\}$ and $\lambda$ such that $\alpha(\lambda)=\frac{1}{\lambda^{p}}$. Then $\frac{1}{\lambda^{p}} \leq \frac{f}{\lambda} \Rightarrow \lambda \geq$ $\left(\frac{1}{f}\right)^{1 / p-1}=\lambda_{0}$, so that

$$
\lambda_{2}=\min \left\{\lambda \geq \lambda_{0}: \alpha(\lambda)=\frac{1}{\lambda^{p}}\right\} .
$$

Let now $\lambda_{1}$ as defined in Lemma 4.3 .

Obviously $\lambda_{1} \geq \lambda_{2}$.

Since $\lambda_{2}$ is the minimum positive $\lambda$ such that $\alpha(\lambda)=1 / \lambda^{p}$, we have from Lemma 4.3 and by continuity reasons that there exists $g:(0,1] \rightarrow \mathbb{R}^{+}$such that

$$
\int_{0}^{1 / \lambda_{2}^{p}} g=\frac{1}{\lambda_{2}^{p-1}}=\frac{1}{\lambda_{2}^{p}} \cdot \lambda_{2}, \int_{0}^{1} g=f, \int_{0}^{1} g^{q}=A, g \leq \psi
$$

and such that

$$
A_{2}=\int_{1 / \lambda_{2}^{p}}^{1} g^{q}=F_{\lambda_{2}}(\alpha)
$$

where $\alpha=\alpha\left(\lambda_{2}\right)=\frac{1}{\lambda_{2}^{p}}$. But then

$$
T_{\lambda_{2}}\left(\alpha\left(\lambda_{2}\right)\right)=S_{\lambda_{2}}\left(\alpha\left(\lambda_{2}\right)\right)=\frac{\Gamma}{\lambda_{2}^{p-q}} .
$$

Since

$$
T_{\lambda_{2}}(\alpha) \leq A_{1}=\int_{0}^{1 / \lambda_{2}^{p}} g^{q} \leq S_{\lambda_{2}}(\alpha)
$$

we have that

$$
F_{\lambda_{2}}\left(\frac{1}{\lambda_{2}^{p}}\right)+\frac{\Gamma}{\lambda_{2}^{p-q}}=A
$$

that is $\lambda_{1}=\lambda_{2}$.

Let now $\lambda=\lambda_{1}=\lambda_{2}$.

Then

$$
F_{\lambda}\left(1 / \lambda^{p}\right)+\frac{\Gamma}{\lambda^{p-q}}=A,
$$

and $\lambda \geq\left(\frac{1}{f}\right)^{1 / p-1}$. For $\mu>\lambda$ and $\beta=\frac{1}{\mu^{p}}$ we have that

$$
\frac{1}{\mu^{p}} \leq \frac{f}{\mu} \text { and } f-\mu \beta \leq 1-\beta^{1-\frac{1}{p}}
$$


Then $F_{\mu}(\beta)=F_{\mu}\left(1 / \mu^{p}\right)$ describes the minimum $L^{q}$-norm value of functions $g$ defined on

$$
\left[\frac{1}{\mu^{p}}, 1\right]=[\beta, 1] \text { for which } \int_{\beta}^{1} g=f-\mu \beta \text { and } g \leq \psi \text {. }
$$

So

$$
F_{\mu}\left(\frac{1}{\mu^{p}}\right)+\frac{\Gamma}{\mu^{p-q}}=\int_{0}^{1} g_{\mu}^{q}
$$

where $g_{\mu}$ is defined such that

$$
g_{\mu}:=\psi,[0, \beta], \quad \int_{\beta}^{1} g_{\mu}^{q}=F_{\mu}\left(\frac{1}{\mu^{p}}\right) \text { and } g_{\mu} \leq \psi .
$$

Since $q>1$ we have that $\mu \mapsto \int_{0}^{1} g_{\mu}^{q}$ is decreasing on $\left(\left(\frac{1}{f}\right)^{1 /(p-1)},+\infty\right)$.

We provide a proof for this right now.

We distinguish two cases

(i) $0<f \leq \frac{p-1}{p} \Rightarrow 0<f-\frac{1}{\mu^{p-1}} \leq \frac{p-1}{p}, \forall \mu \in\left(\left(\frac{1}{f}\right)^{1 /(p-1)},+\infty\right)$.

Then $g_{\mu}$ has the form

$$
\begin{gathered}
g_{\mu}(t)=\left\{\begin{array}{c}
\psi(t), \quad t \in\left(0, \frac{1}{\mu^{p}}\right] \\
c_{\mu}, \quad t \in\left[\frac{1}{\mu^{p}}, 1\right],
\end{array} \quad\right. \text { where } \\
c_{\mu}=\frac{f-\frac{1}{\mu^{p-1}}}{1-\frac{1}{\mu^{p}}}, \quad \text { while } \int_{1 / \mu^{p}}^{1} g_{\mu}^{q}=F_{\mu}\left(1 / \mu^{p}\right) .
\end{gathered}
$$

It is now easy to see that $v>\mu>\left(\frac{1}{f}\right)^{1 /(p-1)} \Rightarrow c_{v}>c_{\mu}$. Additionally $\int_{0}^{1} g_{v}=\int_{0}^{1} g_{\mu}$.

According now to the way that $g_{v}$ and $g_{\mu}$ are defined we obtain, by using Lemma 3.1 that $\int_{0}^{1} g_{\mu}^{q}>\int_{0}^{1} g_{v}^{q}$. That is $\mu \mapsto \int_{0}^{1} g_{\mu}^{q}$ is strictly decreasing on the range $\left(\left(\frac{1}{f}\right)^{1 /(p-1)},+\infty\right)$ for the case $0<f<\frac{p-1}{p}$.

(ii) We consider now the second case $\frac{p-1}{p}<f \leq 1$.

Let $\mu_{0}$ be such that $f-\frac{1}{\mu_{0}^{p-1}}=\frac{p-1}{p}$, so that

$$
\mu_{0}=\left[\frac{1}{\left(f-\frac{p-1}{p}\right)}\right]^{1 /(p-1)}>\left(\frac{1}{f}\right)^{1 /(p-1)} .
$$

Then (A): for every $\left(\frac{1}{f}\right)^{1 /(p-1)}<\mu \leq \mu_{0} \Rightarrow 0<f-\frac{1}{\mu^{p-1}} \leq \frac{p-1}{p}$ : while (B): for $\mu \geq \mu_{0} \Rightarrow$ $f-\frac{1}{\mu^{p-1}} \geq \mu_{0}:(\mathrm{B})$.

Case (A) is worked out as in case (i), and so we see that $\mu \mapsto \int_{0}^{1} g_{\mu}^{q}$ is strictly decreasing on the range $\left(\left(\frac{1}{f}\right)^{1 /(p-1)}, \mu_{0}\right]$. 
Let $\mu$ be as in (B), that is $\mu>\mu_{0}$. Then we define

$$
g_{\mu}(t)=\left\{\begin{array}{cc}
\psi(t), & t \in\left(0, \frac{1}{\mu^{p}}\right] \\
c_{\mu}^{\prime}, & t \in\left(\frac{1}{\mu^{p}}, c_{\mu}\right] \\
\psi(t), & t \in\left(c_{\mu}, 1\right]
\end{array}\right.
$$

where $c_{\mu}$ is defined (see Proposition 3.2) by:

$$
\frac{1}{p} c_{\mu}^{1-\frac{1}{p}}+\left(1-\frac{1}{p}\right) c_{\mu}^{-1 / p}=1-f_{2}=1-\left(f-\frac{1}{\mu^{p-1}}\right)
$$

and $c_{\mu}^{\prime}=\psi\left(c_{\mu}\right)=\left(1-\frac{1}{p}\right) c_{\mu}^{-1 / p}$. From (4.29) we have that

$$
\frac{1}{p} c_{\mu}^{1-\frac{1}{p}}+\left(1-\frac{1}{p}\right) c_{\mu}^{-1 / p}-\frac{1}{\mu^{p-1}}=1-f .
$$

It is not now difficult to see, by differentiating (4.30) with variable $\mu$ that the function $C$ : $\left(\mu_{0},+\infty\right) \rightarrow \mathbb{R}$ defined by $C(\mu)=c_{\mu}$ is strictly decreasing, so according again to Lemma 3.1 we have that $v>\mu \Rightarrow \int_{0}^{1} g_{\mu}^{q}>\int_{0}^{1} g_{v}^{q}$, that is $\mu \mapsto \int_{0}^{1} g_{\mu}^{q}$ is strictly decreasing on $\left(\mu_{0},+\infty\right)$. But it is easy to see that $\mu \mapsto \int_{0}^{1} g_{\mu}^{q}$ is continuous on $\left(\left(\frac{1}{f}\right)^{1 / p-1},+\infty\right)$ so that our assertion is proved.

From the above facts we obtain that for every $\mu>\lambda=\lambda_{1}=\lambda_{2}$ we have that

$$
F_{\mu}\left(\frac{1}{\mu^{p}}\right)+\frac{\Gamma}{\mu^{p-q}}<A
$$

The conclusion is Theorem 4.2 ,

\section{Proof Of Theorem 4.1}

We continue now with the proof of Theorem 4.1

Proof of Theorem 4.1. We will use Theorem 4.1 (i) and (ii).

Let us consider the case $f<\lambda<\lambda_{1}$, so that $\alpha=\alpha(\lambda)=B_{1}(f, A, \lambda)<\frac{1}{\lambda^{p}}$. Of course, we must also have that $\alpha \leq \frac{f}{\lambda}$. We search now for those $\beta \in\left[0, \frac{1}{\lambda^{p}}\right]$ such that $f-\lambda \beta \leq 1-\beta^{1-1 / p}$.

Consider $K$ defined on $\left[0, \frac{1}{\lambda^{p}}\right]$ by $K(\beta)=f-1+\beta^{1-1 / p}-\lambda \beta$. Since $K^{\prime}(\beta)=\frac{p-1}{p} \beta^{-1 / p}-\lambda$, $K$ increasing on $\left[0, \beta_{0}\right]$, decreasing on $\left[\beta_{0}, \frac{1}{\lambda^{p}}\right]$ with maximum value at the point $\beta_{0}$ where $\beta_{0}=\left[\frac{(p-1) / p}{\lambda}\right]^{p}$. Then

$$
K\left(\beta_{0}\right)=f-1+\left[\frac{(p-1) / p}{\lambda}\right]^{p-1} \cdot \frac{1}{p}
$$

which may be positive as well as negative. We first work in case that $K\left(\beta_{0}\right)>0$ and $\frac{p-1}{p}<f \leq$ 1. From the above we have that there exist $\beta_{1}, \beta_{2} \leq \frac{1}{\lambda^{p}}$ with $\beta_{1}<\beta_{2}$ so that $f-\lambda \beta_{i}=1-\beta_{i}^{1-\frac{1}{p}}$ for $i=1,2$ and for $\beta \leq \frac{1}{\lambda^{p}}$ we have that $f-\lambda \beta \leq 1-\beta^{1-\frac{1}{p}}$ if and only if $\beta \in\left[0, \beta_{1}\right] \cup\left[\beta_{2}, \frac{1}{\lambda^{p}}\right]$. With the above hypothesis we prove the following 
Lemma 5.1. For $(f, A) \in D$ such that $A>\mathcal{A}_{f}, f<\lambda<\lambda_{1}$ we have that

$$
\alpha=B_{1}(f, A, \lambda) \in\left[\beta_{2}, \min \left\{\frac{f}{\lambda}, \frac{1}{\lambda^{p}}\right\}\right] .
$$

Proof. Obviously, for $\gamma=\frac{f}{\lambda}, f-\gamma \lambda \leq 1-\gamma^{1-\frac{1}{p}}$ and $\lambda \beta_{2} \leq \beta_{2}^{1-\frac{1}{p}}$ so by means of Proposition 3.1 there exists $\phi:\left[0, \beta_{2}\right] \rightarrow \mathbb{R}^{+}$such that

$$
\int_{0}^{\beta_{2}} \phi=\lambda \beta_{2}, \phi \leq \psi, \int_{0}^{\beta_{2}} \phi^{q}=T_{\lambda}\left(\beta_{2}\right)
$$

Now since $\beta_{2}>\beta_{0}=\left[\frac{p-1 / p}{\lambda}\right]^{p}, T_{\lambda}\left(\beta_{2}\right)=\Delta_{f}\left(\beta_{2}\right)$. We extend now $\phi$ on $[0,1]$ by defining $\phi=\psi$ on $\left[\beta_{2}, 1\right]$. Then since $f-\lambda \beta_{2}=1-\beta_{2}^{1-\frac{1}{p}}$ we have that $\int_{0}^{1} \phi=f$.

By definition now of $\phi$ and $\Delta_{f}\left(\beta_{2}\right)$ the form of $\phi$ must be such that $\int_{0}^{1} \phi^{q}=\mathcal{A}_{f}$. This is true since $\Delta_{f}\left(\beta_{2}\right)$ describes the $L^{q}$-norm of a function of the form

$$
h(t):= \begin{cases}\frac{p-1}{p} c^{-1 / p}, & t \in(0, c] \\ \psi, & t \in[c, 1]\end{cases}
$$

Since then $\phi=\psi$ on $\left[\beta_{2}, 1\right]$ we should have that

$$
\phi(t):=\left\{\begin{array}{lll}
\frac{p-1}{p} c^{-1 / p}, & \text { on } & (0, c] \\
\psi, & \text { on } & {[c, 1]}
\end{array}\right.
$$

almost everywhere and $\int_{0}^{1} \phi=f$ that is $\int_{0}^{1} \phi^{q}=\mathcal{A}_{f}$, by the definition of $\mathcal{A}_{f}$ (see Lemma 3.2). But we remind that $\int_{0}^{\beta_{2}} \phi=\lambda \beta_{2}$. Since now $\int_{0}^{1} \phi^{q}=\mathcal{A}_{f}<A$, and $(f, A) \in D$ we can construct a function $g:(0,1] \rightarrow \mathbb{R}^{+}$such that $g \leq \psi, \int_{0}^{1} g=f, \int_{0}^{1} g^{q}=A$ (by increasing suitably of $\phi$ on an interval of the form $\left(0, c_{1}\right], c_{1} \leq c$ and decreasing it on $\left.\left[c_{1}, 1\right]\right)$ and $\int_{0}^{\gamma} g \geq \gamma \lambda$, for a $\gamma>\beta_{2}$. But then $B_{1}(f, A, \lambda)=\alpha \geq \gamma>\beta_{2}$. Obviously then $\alpha \in\left[\beta_{2}, \min \left\{\frac{f}{\lambda}, \frac{1}{\lambda^{p}}\right\}\right]$.

Consider now the following function defined on

$$
R_{\lambda}: \Delta=\left[\beta_{2}, \min \left\{\frac{f}{\lambda}, \frac{1}{\lambda^{p}}\right\}\right] \rightarrow \mathbb{R}^{+}
$$

with $R_{\lambda}(\beta)=F_{\lambda}(\beta)+S_{\lambda}(\beta), \beta \in \Delta$.

We prove now that $R_{\lambda}$ is increasing on $\Delta$. We are in the case where $\frac{p-1}{p}<f \leq 1$, and $R_{\lambda}$ defined on $\Delta=\left[\beta_{2}, \min \left\{\frac{f}{\lambda}, \frac{1}{\lambda^{p}}\right\}\right]$.

We have that

$$
R_{\lambda}(\gamma)=F_{\lambda}(\gamma)+S_{\lambda}(\gamma), \text { for } \gamma \in \Delta
$$


Here $F_{\lambda}(\gamma)=\int_{\gamma}^{1} g_{\gamma}^{q}$, where $g_{\gamma}$ is defined as

$$
g_{\gamma}(t)= \begin{cases}\left(1-\frac{1}{p}\right) c_{2}^{-1 / p}, & t \in\left(0, c_{2}\right] \\ \psi(t), & t \in\left(c_{2}, \gamma\right] \\ \left(1-\frac{1}{p}\right) c_{1}^{-1 / p}, & t \in\left(\gamma, c_{1}\right] \\ \psi(t), & t \in\left(c_{1}, 1\right]\end{cases}
$$

where $c_{1}, c_{2}$ are such that

$$
\int_{\gamma}^{1} g_{\gamma}=f-\lambda \gamma \text { and } \int_{0}^{\gamma} g_{\gamma}=\lambda \gamma
$$

Then

$$
S_{\lambda}(\gamma)=\int_{0}^{\gamma} g_{\gamma}^{q}, \text { and } R_{\lambda}(\gamma)=\int_{0}^{1} g_{\gamma}^{q}
$$

Easily we see that $c_{2}(\gamma) c_{2}=\left[p \gamma\left(\gamma^{-1 / p}-\lambda\right)\right]^{p /(p-1)}$ and that $\gamma_{1}>\gamma, \gamma_{1}, \gamma_{2} \in \Delta \Rightarrow c_{2}\left(\gamma_{1}\right)<c_{2}(\gamma)$.

Additionally $c_{1}=c_{1}(\gamma)$ satisfies

$$
\Lambda(\gamma)=\frac{1}{p}\left[c_{1}(\gamma)\right]^{1-\frac{1}{p}}+\frac{p-1}{p} \gamma\left[c_{1}(\gamma)\right]^{-1 / p}-\lambda_{\gamma}=1-f .
$$

Differentiating with respect to $\gamma$ we obtain

$$
c_{1}^{\prime}(\gamma) \frac{1}{p} \cdot \frac{p-1}{p}\left[c_{1}(\gamma)\right]^{-1 / p}\left[1-\left(c_{1}(\gamma)\right)^{-1} \gamma\right]=\lambda+\frac{p-1}{p} c_{1}(\gamma)^{-1 / p} .
$$

But $c_{1}(\gamma)>\gamma$, for every $\gamma \in \Delta$.

So from (5.31) we have that $\left(c_{1}(\gamma)\right)$ is an increasing function of $\gamma, \gamma \in \Delta$. Considering again Lemma 3.1 we see that $\gamma<\gamma_{1} \Rightarrow \int_{0}^{1} g_{\gamma_{1}}^{q}>\int_{0}^{1} g_{\gamma}^{q}$ or that $R_{\lambda}\left(\gamma_{1}\right)>R_{\lambda}(\gamma)$.

The other cases are treated in similar ways.

Then we have that $R_{\lambda}(\gamma), \gamma \in \Delta$ is an increasing function.

Let now $\alpha=B_{1}(f, A, \lambda)$ then as we mentioned before, $\alpha \in \Delta$, and of course $T_{\lambda}(\alpha) \leq$ $A-F_{\lambda}(\alpha) \leq S_{\lambda}(\alpha)$ because of Lemma 4.3. If $\alpha<\min \left\{\frac{f}{\lambda}, \frac{1}{\lambda^{p}}\right\}$ and $F_{\lambda}(\alpha)+T_{\lambda}(\alpha)<A$ then since $f-\lambda \alpha<1-\alpha^{1-\frac{1}{p}}$ there exists $\gamma$ such that $\alpha<\gamma<\min \left\{\frac{f}{\lambda}, \frac{1}{\lambda^{p}}\right\}$ such that $F_{\lambda}(\gamma)+T_{\lambda}(\gamma)<A$ and of course

$$
\left.R_{\lambda}(\gamma)=F_{\lambda}(\gamma)+S_{\lambda}(\gamma) \geq A \text { ( } R_{\lambda} \text { is increasing }\right) .
$$

That is

$$
T_{\lambda}(\gamma) \leq A-F_{\lambda}(\gamma) \leq S_{\lambda}(\gamma)
$$

Then Theorem 4.2 gives $B_{1}(f, A, \lambda) \geq \gamma>\alpha$, a contradiction that is if $\alpha=B(f, A, \lambda)<$ $\min \left\{\frac{f}{\lambda}, \frac{1}{\lambda^{p}}\right\}$ we must have that $F_{\lambda}(\alpha)+T_{\lambda}(\alpha)=A$.

Consider now $\lambda_{0}=\left(\frac{1}{f}\right)^{1 / p-1}$ and the function $h: E=\left[f,\left(\frac{1}{f}\right)^{1 /(p-1)}\right] \rightarrow \mathbb{R}^{+}$defined by $h(\lambda)=T_{\lambda}(f / \lambda)$. Notice that for $f \leq \lambda \leq\left(\frac{1}{f}\right)^{1 /(p-1)}$ we have that $\frac{f}{\lambda} \leq \frac{1}{\lambda^{p}}$, so this definition makes sense. Notice also that $\lambda_{1} \geq \lambda_{0}$. 
Then

$$
h(f)=T_{f}(1)=\mathcal{A}_{f}<A \text { and } h\left(\lambda_{0}\right)=\Gamma \frac{1}{\lambda_{0}^{p-q}}=\Gamma f^{p-q / p-1} \geq A .
$$

Consider now all $\lambda \in E$ such that

$$
h(\lambda)=T_{\lambda}(f / \lambda) \leq A
$$

Then

$$
T_{\lambda}(f / \lambda) \leq A=A-F_{\lambda}(f / \lambda) \leq \Gamma f^{p-q / p-1}=S_{\lambda}(f / \lambda)
$$

so in view of Corollary $4.2, B_{1}(f, A, \lambda)=\frac{f}{\lambda}$.

For $\lambda \in E \cap\{\lambda: h(\lambda)>A\}$ we have by the above comments that

$$
B_{1}(f, A, \lambda)=\sup \left\{\alpha \in \Delta: F_{\lambda}(\alpha)+T_{\lambda}(\alpha)=A\right\} .
$$

Additionally the same equality holds for $\lambda$ such that $\left(\frac{1}{f}\right)^{1 / p-1}<\lambda \leq \lambda_{1}$.

So we found $B_{1}(f, A, \lambda)$ as $f / \lambda$ or a maximum root of an equation in case where $\lambda \in\left[f, \lambda_{1}\right]$, $K\left(\beta_{0}\right)>0, \frac{p-1}{p}<f \leq 1$.

The case $K\left(\beta_{0}\right)=0$ is worked out in the same way where we replace $\beta_{2}$ by $\beta_{0}$, while the case $K\left(\beta_{0}\right)<0$ is worked out for

$$
\Delta=\left[0, \min \left\{\frac{f}{\lambda}, \frac{1}{\lambda^{p}}\right\}\right]
$$

Analogous results are obtained when $0<f \leq \frac{p-1}{p}$ where

$$
\Delta=\left[0, \min \left\{\frac{f}{\lambda}, \frac{1}{\lambda^{p}}\right\}\right]
$$

since then

$$
f-\lambda \beta \leq \frac{p-1}{p}(1-\beta) \leq 1-\beta^{1-\frac{1}{p}} \text { for every } \beta \leq \frac{1}{\lambda^{p}}
$$

We prove now the following fact.

The function $h(\lambda)=T_{\lambda}(f / \lambda)$ defined on $E=\left[f,\left(\frac{1}{f}\right)^{1 /(p-1)}\right]$ as above is strictly increasing.

We consider again two cases.

i) $0<f<\frac{p-1}{p}$. Then for $f<\lambda<\left(\frac{1}{f}\right)^{1 /(p-1)} \cdot\left(\frac{p-1}{p}\right)^{p /(p-1)}$ we have that $\frac{f}{\lambda} \leq\left[\frac{(p-1) / p}{\lambda}\right]^{p}$ so that $h(\lambda)=\lambda^{q-1} f$, which is certainly strictly increasing on this range.

As for the range $\left(\frac{1}{f}\right)^{1 /(p-1)}\left(\frac{p-1}{p}\right)^{p /(p-1)}<\lambda<\left(\frac{1}{f}\right)^{1 / p-1}$ we have that $\left[\frac{(p-1) / p}{\lambda}\right]^{p} \leq \frac{f}{\lambda} \leq \frac{1}{\lambda^{p}}$, so that

$$
\begin{aligned}
& h(\lambda)=\Delta_{\lambda_{\alpha}}(\alpha)=\int_{0}^{\alpha} g_{\lambda}^{q}, \quad \text { where } \\
& g_{\lambda}(t)=\left\{\begin{array}{cc}
\frac{p-1}{p} c_{2}^{-1 / p}, & t \in\left(0, c_{2}\right] \\
\psi(t), & t \in\left(c_{2}, \alpha\right]
\end{array}\right.
\end{aligned}
$$

where $c_{2}<\alpha$ is such that $\int_{0}^{\alpha} g_{\lambda}=\lambda \alpha=\lambda \frac{f}{\lambda}=f$. 
We prove that $h(\lambda)$ is increasing on this range also. Since it is continuous on all $E$ we obtain the desired result. Easily

$$
c_{2}(\lambda)=c_{2}=\left\{p\left[\left(\frac{f}{\lambda}\right)^{1-\frac{1}{p}}-\lambda \alpha\right]\right\}^{p / p-1} .
$$

So $c_{2}(\lambda)$ is decreasing on the above range and thus by using Lemma 3.1 we obtain what we wanted to prove.

ii) The second case $\frac{p-1}{p}<f \leq 1$ is easier to handle because $h(\lambda)=T_{\lambda}(f / \lambda)$ is defined in a certain way in all $E$, as we have done in the case i) (second part).

We need to say a few comments for the case $f<\lambda<\frac{p-1}{p}$.

In this case we have the same results with $F_{\lambda}(\alpha), T_{\lambda}(\alpha)$ given by:

$$
F_{\lambda}(\alpha)=\frac{(f-\lambda \alpha)^{q}}{(1-\alpha)^{q-1}} \text { and } T_{\lambda}(\alpha)=\lambda^{q} \alpha
$$

and then

$$
B_{1}(f, A, \lambda)=\sup \left\{\alpha \in \Delta: \frac{f-\lambda \alpha)^{q}}{(1-\alpha)^{q-1}}+\lambda^{q} \alpha=A\right\}
$$

for $\Delta=\left[\beta_{2}^{\prime}, \min \left\{\frac{f}{\lambda}, \frac{1}{\lambda^{p}}\right\}\right]$ for some suitable constant $\beta_{2}^{\prime}$.

This finishes the proof of Theorem 4.1 .

Remark 5.1. i) The case where $A=\mathcal{A}_{f}$ can be worked out separately because there exists essentially a unique function $g:[0,1] \rightarrow \mathbb{R}^{+}$such that $\int_{0}^{1} g=f, \int_{0}^{1} g^{q}=A, g \leq \psi$.

ii) We have that $B(f, A, \lambda)=B_{1}(f, A, \lambda)$, for $A \neq f^{q}$ as mentioned in the beginning of this section. This is true of course for $\lambda \geq \lambda_{1}$, that is for $\lambda$ such that $\alpha(\lambda)=B_{1}(f, A, \lambda)=\frac{1}{\lambda^{p}}$.

Now for $\lambda<\lambda_{1}$ let $\alpha=B_{1}(f, A, \lambda)$. Then there exists $g:[0,1] \rightarrow \mathbb{R}^{+}$such that $\int_{0}^{1} g=f$, $\int_{0}^{1} g^{q}=A, \int_{0}^{\alpha} g=\alpha \lambda, g \leq \psi$. Then it is easy to see that for every $\varepsilon>0$ small enough we can change $g$ to $g_{\varepsilon}$ in a way that

$$
\int_{0}^{\alpha-\varepsilon} g_{\varepsilon} \geq(\alpha-\varepsilon) \lambda, \quad \int_{0}^{1} g_{\varepsilon}=f, \quad \int_{0}^{1} g_{\varepsilon}^{q}=A+\delta_{\varepsilon}, \quad\left\|g_{\varepsilon}\right\|_{p, \infty}=\frac{p-1}{p}
$$

and $\delta_{\varepsilon} \rightarrow 0$ as $\varepsilon \rightarrow 0^{+}$. This using continuity arguments gives $B(f, A, \lambda)=\alpha$.

iii) Notice the continuity of the function as calculated on Theorem 4.2, at the point $\lambda=\lambda_{1}$. As a matter of fact $\delta$ is such that $F_{\lambda_{1}}(\delta)+T_{\lambda_{1}}(\delta)=A$. But $\lambda_{1}$ is such that

$$
F_{\lambda_{1}}\left(\frac{1}{\lambda_{1}^{p}}\right)+\frac{\Gamma}{\lambda_{1}^{p-q}}=A, \text { and } \frac{\Gamma}{\lambda_{1}^{p-q}}=S_{\lambda_{1}}\left(\frac{1}{\lambda_{1}^{p}}\right)=T_{\lambda_{1}}\left(\frac{1}{\lambda_{1}^{p}}\right) \text {. }
$$

So that

$$
F_{\lambda_{1}}(\delta)+T_{\lambda_{1}}(\delta)=F_{\lambda_{1}}\left(\frac{1}{\lambda_{1}^{p}}\right)+T_{\lambda_{1}}\left(\frac{1}{\lambda_{1}^{p}}\right)=A,
$$

which in view of Remark iii) above, gives $\delta=\frac{1}{\lambda_{1}^{p}}$.

Theorem 1.2 is now an immediate consequence. 


\section{REFERENCES}

[1] C. Bennet, R. Sharpley, Interpolation of Operators Academic Press.

[2] D. L. Burkholder, Martingales and Fourier Analysis in Banach spaces, C.I.M.E. Lectures (Varenna (Como), Italy, 1985), Lecture Notes in Mathematics 1206(1986), 61-108.

[3] D. L. Burkholder, Boundary value problems and sharp inequalities for martingale transforms, Ann. of Prob. 12(1984), 647-702.

[4] A. D. Melas, The Bellman functions of dyadic-like maximal operators and related inequalities, Adv. Math. 192(2005) 310-340.

[5] A. D. Melas, E. Nikolidakis, Dyadic-like maximal operators on integrable functions and Bellman functions related to Kolmogorov's inequality, Transactions of the American Mathematical Society, vol. 362, No 3, March 2010, papes: 1571-1596.

[6] A. D. Melas, E. Nikolidakis, On weak type inequalities for dyadic maximal functions, J. Math. Anal, Appl. (2008) 404-410.

[7] E. N. Nikolidakis, Extremal problems related to maximal dyadic-like operators, J. Math. Anal. Appl. $369(2010) 377-385$.

[8] G. Wang, Sharp maximal inequalities for conditionally symmetric martingales and Brownian motion, Proc. Amer. Math. Soc. 112(1991) 579-586. 